\title{
Charge Transfer Reactions in Electrochemical and Chemical Processes
}




\section{Charge Transfer Reactions in \\ Electrochemical and Chemical Processes}

\section{I. Krishtalik}

Institute of Electrochemistry

Academy of Sciences of the USSR

Moscow, USSR

Translated by

R. S. Wadhwa

and

N. V. Deineko

Translation Edited by

R. G. Compton

Physical Chemistry Laboratory

Oxford University

Oxford, England 


\title{
Library of Congress Cataloging in Publication Data
}

Krishtalik, Lev Isaevich.

Charge transfer reactions in electrochemical and chemical processes.

Translation of: Élektrodnye reaktsii.

Bibliography: $p$.

Includes index.

1. Electrodes. I. Title.

QD571.K8313 1986

ISBN-13: 978-1-4684-8720-6

$541.3^{\prime} 724$

e-ISBN-13: 978-1-4684-8718-3

86-8866

DOI: $10.1007 / 978-1-4684-8718-3$

This volume is based on the original Russian edition, published in 1979

by Nauka, Moscow, and has been extensively rewritten and updated.

This translation is published under an agreement with the

Copyright Agency of the USSR (VAAP)

\section{ÉLEKTRODNYE REAKTSII. MEKHANIZM ÉLEMENTARNOGO AK'TA}

\author{
L. I. Krishtalik
}

(c) 1986 Consultants Bureau, New York

Softcover reprint of the hardcover 1st edition 1986

A Division of Plenum Publishing Corporation

233 Spring Street, New York, N.Y. 10013

All rights reserved

No part of this book may be reproduced, stored in a retrieval system, or transmitted in any form or by any means, electronic, mechanical, photocopying, microfilming, recording, or otherwise, without written permission from the Publisher 
Dedicated to the memory of

Aleksandr Naumovich Frumkin 


\section{Preface}

The mechanism of an elementary act is undoubtedly one of the most fundamental problems of chemical and, in particular, electrochemical kinetics. Although this problem has fascinated scientists for quite a long time, it was only in the late fifties and early sixties that it began to be actively investigated for charge transfer reactions. Owing to the development of new methods in the analysis of this problem, significant advancements were made in theoretical as well as experimental studies. These investigations showed that the physical mechanism of charge transfer in all processes including heterogeneous electrochemical and homogeneous chemical and biochemical processes is basically the same. Hence, the results obtained in the field of electrochemical kinetics are relevant to the understanding of homogeneous chemical reactions as well.

This book endeavors to summarize the results of investigations carried out during the last two decades. It is based on the author's monograph "Electrode Reactions: The Mechanism of an Elementary Act" (Nauka, 1979). As compared to the first version, the book has been considerably revised and enlarged not only to include a large body of data published between 1978 and 1982, but also to analyze in detail the links between electrochemical and homogeneous, in particular enzymatic, kinetics. As a result, a new chapter has been added to the book. The change in the title reflects the fact that the material contained in the book is not restricted to an investigation of purely electrochemical problems.

The first chapter is devoted to the phenomenological theory of an elementary act. It considers the Bronsted-Polanyi relation and some of its corollaries, as well as real and ideal activation energies and preexponential factors of electrochemical reactions. 
Special attention has been paid to the theory of activationless and barrierless processes.

The second chapter deals with experimental verification of the theory, i.e. the study of barrierless discharge of hydrogen ions. The main body of the experimental data is connected with the evolution of hydrogen at a mercury and, to a lesser extent, a silver cathode. At the end of this chapter, there is a brief summary of such questions concerning the theory of barrierless processes whose consideration is beyond the framework of a purely phenomenological description.

The third chapter aims at a systematic analysis of the modern quantum-mechanical theory of charge transfer reactions. An attempt has been made to give a complete description of the physical principles of the theory almost without resorting to its detailed mathematical formalism. Such a form of presentation is guided by the author's endeavor to enable the reader to use the results of this theory without any need to acquire a special theoretical background in quantum mechanics.

The main difference between the new and the old theories of an elementary act lies in the interpretation of the mechanism of proton transfer, in particular, of the discharge of proton donors. This question is elucidated in the fourth chapter where a considerable amount of experimental data has been given to demonstrate the advantage of the new, quantum-mechanical model of an elementary act.

The electrode processes associated with the transfer of heavy particles are characterized by a number of peculiar features. They are described in the fifth chapter which is mainly devoted to theoretical and experimental studies of quasibarrierless and quasiactivationless processes. In particular, it is shown that one of the most important reactions in the electrochemical industry, viz. the anodic evolution of chlorine, is a quasibarrierless process.

The sixth chapter considers the possibilities of solving a number of old and new problems in electrochemical kinetics, in particular the hydrogen evolution reaction is considered using the methods of the theory of an elementary act of electrode processes.

Finally, the seventh chapter is devoted especially to an investigation of problems concerning homogeneous chemical kinetics, and above all, proton transfer reactions, as well as the application of new concepts to the analysis of enzymatic reactions.

The sections marked by asterisks can be omitted by the readers not interested in a detailed analysis of the problem. 
The author wishes to express his profound gratitude to all his colleagues who took part in experimental studies presented in this book. Special mention must be made about the assistance rendered by V. I. Bystrov, R. G. Erenburg, L. A. Khanova, D. E. Khoshtariya, D. V. Kokoulina, Z. A. Rotenberg, G. E. Titova, V. V. Topolev, and V. M. Tsionsky. Experimental studies on the problems of an elementary act were carried out side by side with the theoretical investigations, and it is a pleasure to place on record our fruitful collaboration with the staff of the theoretical department of the Institute of Electrochemistry, and above all with R. R. Doganadze and A. M.

Kuznetsov. Besides the persons listed above, Yu. V. Pleskov and Yu. M. Polukarov have also read the manuscript and made valuable observations.

During the course of his investigations on the mechanism of an elementary act, the author greatly benefited from the attention and continuous encouragement from Academician A. N. Frumkin whose inspiration has been responsible for the all-round development of this field. It is with a profound sense of reverence and indebtedness that I dedicate this book to his memory.

L. I. Krishtalik 


\section{Introduction}

Reactions involving charge transfer form one of the broadest classes of chemical transformations. They include such important processes as redox reactions (in the simplest case, electron transfer), acid-base transformations (proton transfer), electrolytic dissociation, and many others. A special class of heterogeneous processes of charge transfer includes electrode reactions associated with a charge transfer through the interface between electronic and ionic conductors.

It was assumed for a long time that the charge transfer (for example, discharge of an ion) is an extremely rapid process, and the measured rate of reaction is completely determined by the other stages of the process, for example, by subsequent chemical reactions between neutral particles. Historically, this idea was put forward at the time of creation of the ionic theory, when rapid ("practically instantaneous") transformation of ions into undissociated products was considered as one of the proofs of electrolytic dissociation. As a matter of fact, the rates of a very large number of chemical reactions involving charge transfer were too high to be measured with the help of traditional methods of chemical kinetics. Hence it seemed natural to attribute the comparatively low rates of some electrode reactions to secondary causes.

However, the finite rate of electrochemical processes itself is the dominating concept of electrochemical kinetics at present. This concept, developed and substantiated mainly by Academician A. N. Frumkin and his school, was found to be applicable to different kinds of electrode reactions, from simple recharging of ions to complex multistage processes[1]. 
By considering the kinetic substantiation of the Nernst equation, Audubert[2] and Butler[3] were the first to formulate the semiquantitative concepts concerning the effect of electrode potential on the rate of an electrochemical process. In its present form, the concept of slow discharge was put forward by Erdey-Gruz and Volmer[4]. Their approach was based on a consideration of the effect of potential on the activation energy of an electrode process and led to the introduction of a measure of this effect, viz. the transfer coefficient a.

At first, however, it seemed that the concept of slow discharge of hydrogen ions (on which process many fundamental investigations of electrochemical kinetics were undertaken) was in irreconcilable contradiction with experiment, and above all with the data which indicate that the hydrogen overpotential is independent of the acid concentration in solution. This contradiction was removed in the classical work by Frumkin[5], where the effect of the double-layer structure on the discharge rate was taken into account. Since then, numerous studies have resulted in a reliable substantiation of the concept of the finite rate of charge transfer through the electrodesolution interface.

Naturally, the next stage in the investigation of electrode processes is the statement of the problem concerning the mechanism of an elementary act of charge transfer. Of course, this question fascinated scientists from the very onset of the theory of slow discharge. As early as the thirties, Gurney[6], Horiuti and Polanyi[7], and others put forward a number of important ideas which continue to be valid even today. Nevertheless the main problem in the investigations carried out between the thirties and the fifties was to elucidate the sequence of stages of various electrode reactions and to rigorously substantiate the fact that in a number of cases the charge transfer is the stage that determines the rate of the process as a whole. On the basis of these experimental studies and advances in theoretical kinetics and in condensed state physics, scientists whose works will be presented below developed modern concepts about the molecular pattern of an elementary act of electrode processes and in general reactions accompanied by a transfer of charged particles. This pattern differs in many respects from the one that was accepted until recently and described in all textbooks on chemical kinetics and electrochemistry.

Naturally, the clearest picture of the processes taking place during an elementary act is obtained by considering the mechanism on a molecular scale. However, any model used for a quantitative treatment of the problem as a rule introduces certain simplifications, thus making it difficult to obtain an exact interpretation of the experimental data. Hence, the molecular approach is successfully supplemented by the phenomenological description introduced by Frumkin in his well-known paper[8] establishing the connection 
between the Tafel equation and the Brфnsted relation. The phenomenological (sometimes semiphenomenological) approach made it possible to elucidate some general regularities and to predict phenomena which were subsequently observed experimentally. An important advantage of the phenomenological analysis lies in that it enables us to clarify the meaning of certain quantities measured in electrochemical experiments and to find a method for comparing them with any theoretical mode1, as was done by Temkin $[9,10]$.

In this book, both approaches will be used while considering an elementary act of charge transfer reactions. It should be noted that these two approaches overlap to a certain extent. Thus, the phenomenological description includes at various stages some model concepts, while the molecular approach uses a phenomenological description for certain important phenomena, for example, the behavior of the solvent. Of late, a combination of these two approaches has led to a number of important results. In particular, it has provided a substantiation for the concept of quasibarrierless and quasiactivationless reactions. Processes of this type clearly demonstrate the importance of understanding the mechanism of an elementary act for a correct analysis of the kinetics of multistage reactions.

Phenomenologically, as well as from the point of view of the molecular concept of an elementary act, there is much in common between electrode processes and homogeneous charge transfer reactions. However, electrochemical methods were found to be more useful for the analysis of some theoretical problems. Indeed, the dependence of the activation energy on potential makes it possible to vary this parameter over a wide range and thus carry out reactions that can proceed only electrochemically, for example proton transfer to a base (metal) whose binding energy with hydrogen is four times smaller than the corresponding value for the proton donor. Another advantage of the electrochemical kinetics, which we shall be discussing in detail in this book, lies in the possibility of eliminating the effect of certain thermodynamic factors, such as the solvation energy of ions, on the kinetics of a process. All these circumstances indicate that many results of electrochemical investigations can be used for analyzing a wide range of chemical and biochemical charge transfer processes. In particular, we shall endeavor to demonstrate the fruitfulness of such an approach in the last chapter of this book. 


\section{Contents}

1. THE PHENOMENOLOGICAL THEORY OF AN ELEMENTARY ACT

1.1. The Bronsted Relation and the Activation

Energy of Electrode Reactions

1.2. The Temperature Dependence of the Rate of an Electrode Reaction

1.3. The Chemical Potential of an Electron, Absolute Potential Drop, and Solvation Energy in Electrochemical Kinetics

1.4. The Bronsted Relation and the Activity Coefficient of an Activated Complex

1.5. Barrierless and Activationless Processes

1.6. Kinetic Equations for Discharge

2. BARRIERLESS DISCHARGE IN CATHODIC HYDROGEN EVOLUTION

2.1. The Discharge of Hydronium Ions at a

Mercury Cathode

2.2.* Hydrogen Evolution in the Presence of

Tetraalkylammonium Ions. Discharge of Undissociated Acid Molecules

2.3. The Activity Coefficient of an Activated Complex

2.4. The Temperature Dependence of the Rate of a Barrierless Discharge

2.5.* Evolution of Hydrogen at a Silver Cathode

2.6.* Evolution of Hydrogen at Other Cathodes

2.7. Some Theoretical Problems

3. THE QUANTUM-MECHANICAL THEORY OF AN ELEMENTARY ACT

3.1. Introduction

3.2. An Ion in a Polar Solvent 
3.3. The Elementary Act of Electron Transfer

3.4. Adiabatic and Nonadiabatic Transitions

3.5. Quantum and Classical Degrees of Freedom.

4. EXPERIMENTAL VERIFICATION OF THE THEORIES OF AN ELEMENTARY ACT OF PROTON DONOR DISCHARGE

4.1. Statement of the Problem

4.2. Barrierless Discharge. Preexponential

Factor for Barrierless and Ordinary Processes

4.3. Kinetic Isotope Effect for Metals with High Hydrogen Overpotentials

4.4. Effect of the Nature of the Electrode Metal on the Preexponential Factor and the Kinetic Isotope Effect

4.5. Effect of the Potential and the Nature of Proton Donors on the Preexponential Factor and the Kinetic Isotope Effect

4.6. Effect of the Nature of the Solvent on the Elementary Act of the Discharge of Proton Donors

4.7. Conclusion

5. DISCHARGE OF HEAVY IONS. QUASIBARRIERLESS AND QUASIACTIVATIONLESS PROCESSES

5.1. The Elementary Act of a Process Accompanied by the Motion of Heavy Particles

5.2. The Theory of Quasibarrierless and Quasiactivationless Processes

5.3. Anodic Evolution of Chlorine at a Graphite Electrode

5.4.* The Chlorine Evolution Reaction at Ruthenium Dioxide-Titanium Dioxide Anodes

5.5.* The Kinetics of Oxidation of an Azide Ion at a Platinum Anode

5.6. On the Possibility of Other Quasibarrierless and Quasiactivationless Electrode Reactions

6. MECHANISM OF AN ELEMENTARY ACT AND THE KINETICS OF THE CATHODIC EVOLUTION OF HYDROGEN

6.1. Kinetic Isotope Effect and $\psi_{1}$-potential. Localization of a Discharging Ion

6.2.* Kinetic Isotope Effect. Discrimination of the $\psi_{1}$-effect and the Action of Other Factors 
6.4. Mechanism of Amalgam Decomposition

6.5. Hydrogen Evolution at Iron, Chromium and Manganese during Their Chemical Selfdissolution and Cathodic Polarization

6.6. The Ratio of Ortho- and Para-forms of Hydrogen in an Electrolytic Gas

6.7. Cathodic Hydrogen Evolution in Nonaqueous

6.8. Electrode Reactions Involving Atomic Hydrogen Generated Through Interaction with Photoelectrons

7. KINETICS OF HOMOGENEOUS AND ENZYMATIC REACTIONS INVOLVING CHARGE TRANSFER

7.1. Kinetic Isotope Effect in Homogeneous Proton Transfer Reactions

7.2. Medium Reorganization Energy in Proton Transfer Reactions. Comparison of Homogeneous and Electrode Reactions

7.3. Simultaneous Transfer of Two Charges. Coupling of Endoergic and Exoergic Reactions

7.4. Proton Transfer in Enzymatic Hydrolysis

7.5. On the Theory of Enzymatic Charge Transfer Reactions 